\title{
Reformas y reformadores. Hacia el desmantelamiento de los Colegios y la Universidad de Alcalá a partir del Colegio de las Santas Justa y Rufina o de los Sevillanos
}

\author{
Reforms and Reformers. Towards the Dismantling \\ of the Colleges and the University of Alcalá Starting \\ from the College of Saints Justa and Rufina also Called \\ the College of the Sevillians
}

\author{
Manuel Casado Arboniés \\ Universidad de Alcalá-UNED-ACISAL \\ Emiliano Gil Blanco \\ Universidad de San Francisco de Quito (Ecuador)-ACISAL \\ Francisco Javier Casado Arboniés* \\ ACISAL
}

DOI: http://dx.doi.org/10.20318/cian.2016.3438

$\begin{array}{ll}\text { Recibido: } & 31 / 05 / 2016 \\ \text { Aceptado: } & 15 / 10 / 2016\end{array}$

Resumen: Dos son las reformas universitarias que influyeron en el desarrollo y continuidad de la Universidad de Alcalá, en general, y del Colegio de las Santas Justa y Rufina o de los Sevillanos, en particular. La primera de ellas es las que realizó el visitador García de Medrano entre 1663 y 1666, que supuso la reorganización de sus colegios menores, su patrimonio y sus constituciones. Esta refor-
Abstract: There were two main university reforms that influenced on the development and continuity of the University of Alcala (in general) and of the College of Saints Justa and Rufina also known as the College of the Sevillians (in particular). The first reform was made by Garcia Medrano between 1663 and 1666, which led to the reorganization of smaller schools, its assets and its policies.

*manuel.casado@ya.com - emigilblanco@gmail.com - fjca27@gmail.com 
ma no afectó al Colegio de las Santas Justa y Rufina o de los Sevillanos. La segunda fue la de Pedro Díaz de Rojas, en 1775, dentro de la reforma de las Universidades en España y sus colonias que el rey Carlos III puso en marcha. Para la Universidad de Alcalá esta reforma supuso el inicio de su declive y desaparición. Las causas del declive de la universidad hispana recayeron, según los pensadores ilustrados, en la Compañía de Jesús por intentar controlar la educación en todos sus niveles y en la propia institución colegial que representaba un espíritu corporativista para repartirse los puestos de gobierno y administración, tanto civil como eclesiástica. En 1842 sus colegios fueron cerrados y sus bienes fueron traspasados a la Universidad Central de Madrid.

Palabras clave: universidad, reforma, Alcalá, colegios, García de Medrano, Díaz de Rojas.
This reform did not affect the College of Saints Justa and Rufina (the College of the Sevillian). The second reform was made by Pedro Díaz de Rojas in 1775, and was part of the university reform that King Carlos III began in Spain and its colonies. For University of Alcala this reform marked the beginning of its decline and disappearance. According to Enlightenment thinkers, the causes that led to the decline of this Hispanic university fell upon the Society of Jesus (Compañía de Jesús) for trying to control all levels of education and in particular for the corporatist spirit of its own collegial institution that distributed government and management positions alike, both civil and ecclesiastical. In 1842 their schools were closed and their assets were transferred to the Central University of Madrid.

Keywords: university, reform, Alcalá, colleges, García de Medrano, Díaz de Rojas.

\section{Introducción}

El Colegio de las Santas Justa y Rufina, dentro del conjunto colegial de la Universidad de Alcalá, no podía escapar al devenir del mundo universitario español en el siglo XVII y XVIII, bien estudiado, sobre todo a partir de 1770, cuando comience la profunda reforma universitaria promovida por Carlos III.

Una vez más, para el Colegio Mayor de San Ildefonso y Universidad de Alcalá, con toda su pléyade de colegios menores de distinta naturaleza y condición, topamos con el vacío historiográfico ${ }^{1}$, ya que no contamos con ningún estudio monográfico y sabemos tan sólo de alguna aproximación parcial al tema. Sin embargo, los distintos autores coinciden a la hora de señalar el "carácter decadente generalizado" que caracteriza al menos a los primeros cincuenta años del siglo XVIII en el ámbito universitario, y lo que ocurre en el Colegio de las Santas Justa y Rufina es un vivo ejemplo de ello.

${ }^{1}$ Una aproximación a esta historiografía la encontramos en Casado, Manuel, "Los colegios menores seculares de patronato eclesiástico de Universidad de Alcalá. Líneas de investigación". (En Rodríguez-San Pedro, Luis E. y Polo, Juan L. (Eds.): Universidades clásicas de la Europa Mediterránea: Bolonia, Coimbra y Alcalá. Salamanca. Miscelánea Alfonso IX, 2005). Casado, Manuel, Alonso, Pedro M.: "Colegios menores seculares de patronato eclesiástico y naciones estudiantiles en la Universidad de Alcalá de Henares (siglos XVI-XVII)". Revista de Ciencias de la Educación: Órgano del Instituto Calasanz de Ciencias de la Educación, 212 (2007). Valle, José L. (Coord.): Colegios Menores Seculares de la Universidad de Alcalá. (Universidad de Alcalá. Alcalá de Henares. Institución de Estudios Complutenses. 2012). 


\section{La visita de García de Medrano}

Pero antes, entre 1663 y 1666, ya se había producido la intervención en la Universidad de Alcalá del visitador García de Medrano, doctor en cánones y catedrático de Sexto, que entonces era Consejero de Castilla y de la Inquisición, y que había sido colegial del Mayor de San Bartolomé de Salamanca. Una visita y reforma de graves repercusiones por la amplitud de sus planteamientos $^{2}$ y el tiempo que abarca la tarea reformadora, cuatro años ${ }^{3}$, para verificar el grado de cumplimiento de las constituciones y solicitar del Consejo de Castilla la reforma de todo aquello que fuera necesario.

Conocemos la crónica de la visita, llena de datos sumamente significativos, pero que no se limitó de manera exclusiva al Colegio Mayor de San Ildefonso y a los colegios menores, incluido el de las Santas Justa y Rufina, sino que se extendió también al conjunto de propiedades ubicadas en Alcalá de Henares y su tierra, pertenecientes a la Universidad de Alcalá, porque se dice expresamente del reformador que lo es, "de este Collegio Maior y Collegio de San Pedro y San Pablo, que esta junto a el, y las casas de los collegios menores, y otras que dependen del dicho Collegio Maior".

La visita consistió en levantar una "información secreta" del Colegio Mayor de San Ildefonso, realizada por el propio visitador Medrano, conteniendo el compendio de preguntas formuladas a los colegiales mayores, así como su posterior respuesta, comenzando por el colegial más antiguo. Lle-

${ }^{2}$ Gil, Ángel. "Consideraciones en torno al reformismo universitario castellano en el siglo XVII". (En Actas del Congreso de Jóvenes Historiadores y Geógrafos. Tomo II. Madrid. 1990). Gil, Ángel: "Reformas en los colegios menores cisnerianos de la Universidad de Alcalá de Henares durante el siglo XVII". (En Actas del III Encuentro de Historiadores del Valle del Henares. Guadalajara. 1992). Gil, Ángel: "Visitas y reformas de la Universidad de Alcalá en el siglo XVII". Anales Complutenses, IV-V (1992-1993). Gil, Ángel: "Reformas de los colegios menores no cisnerianos de la Universidad de Alcalá durante el siglo XVII". Anales Complutenses, VIII (1996). Gil, Ángel: "Reformas de los colegios menores". (En Valle, José L. (Coord.): Colegios menores seculares de la Universidad de Alcalá. Alcalá de Henares. Institución de Estudios Complutenses. 2012).

${ }^{3}$ Martín, Mariano. Estado de la Universidad de Alcalá [1805]. (Madrid. Instituto Antonio de Nebrija de Estudios sobre la Universidad. Editorial Dykinson. 1999). Este informe fecha la Visita de García de Medrano entre los años 1662 y 1663.

4 "Reformaçion de el Colegio maior de San Ilefonso y Universidad de Alcala, de Henares, que resulta de la visita que hiço el S. D. D. Garçia de [Medrano] de el Consejo de su Magestad, en el Real de Castilla, Visitador y Reformador general de el dicho Colegio, maior, y Universidad; Año de 1663". AHN. Madrid. Consejos, Leg. 5427, LXV, 16. Contiene la práctica totalidad de la documentación generada durante la visita de García de Medrano; y se completa con un libro de la Sección de Universidades. A.H.N. Madrid. Universidades, lib. 525-F. 
var dos cuadernos, uno de descargos de la visita del Colegio Mayor de San Ildefonso, y otro de comprobación de la "información secreta". Y el grueso de la propia visita realizada al Colegio Mayor de San Ildefonso y a los colegios menores adscritos a la Universidad de Alcalá, encontrándose fraudes y desviaciones constitucionales y revisándose sus bienes muebles e inmuebles. Se redactaron las distintas recomendaciones realizadas por el visitador como resultado de su actividad, que junto al texto original y manuscrito de las Constituciones de la Universidad de Alcalá fue enviado al Consejo de Castilla para su aprobación.

A finales de 1662, Medrano comenzó a inspeccionar la totalidad de los edificios colegiales y otras propiedades de la universidad, empezando por el Mayor de San Ildefonso y el franciscano de San Pedro y San Pablo, pero siendo visitados, con resultados positivos, una serie de colegios menores, tales como el de Irlandeses, el de Vizcaínos, el de San Jerónimo de Lugo, el de San Clemente de los Manchegos, el Colegio de León, etc. El Colegio de las Santas Justa y Rufina, tampoco se vio afectado directamente, mientras que otros desaparecieron mediante un proceso de fusiones y agregaciones.

En el terreno institucional, la reforma de Medrano destaca entre otras realizadas a lo largo del siglo XVII, porque se convirtió en norma legal preferente para la Universidad de Alcalá y sus colegios, así como para otras de sus propiedades y para los propios aforados universitarios, hasta las reformas de finales del siglo XVIII. El propio Francisco Pérez Bayer reconocía sobre ella que, "la suma de toda la legislación, o Derecho escrito, por donde se govier$n a^{\prime \prime}$, el Colegio Mayor de San Ildefonso y toda la Universidad de Alcalá eran, y por ese orden, " $1{ }^{\circ}$ las Constituciones del Fundador: $2^{\circ}$ El Reforme de Medrano: 3 la Visita de 1750: 4o la Reformacion de los quatro Ministros de 1753"5.

El visitador Medrano presentó al Consejo de Castilla los documentos que recogían las importantes reformas que tanto necesitaba la Universidad de Alcalá y sus Constituciones. Estas no fueron autorizadas, aunque eran perfectamente necesarias y asumibles por la Universidad de Alcalá, por el propio Consejo de Castilla y por la Corona, a quienes beneficiarían los cambios. Otra cosa es lo que luego opinaron los miembros del Consejo que vetaron varias de las propuestas del Visitador. En todo caso el resultado de la amplia reforma pretendida por Medrano se vio mermado en muchos de sus objetivos.

\footnotetext{
${ }^{5}$ Pérez, Francisco. Por la libertad de la literatura española. Estudio preliminar de Antonio Mestre Sanchís. (Alicante. Instituto de Cultura Juan Gil Albert. Diputación de Alicante. 1991). Pérez, Francisco: Diario histórico de la reforma de los seis Colegios Mayores de Salamanca, Valladolid y Alcalá. (Valencia. Generalitat Valenciana. 2002).
} 
A pesar de todo, la visita de Medrano no se basó sólo, como tantas otras visitas, en supervisar sin más el estado legal de la comunidad universitaria, analizando el cumplimiento de sus constituciones, o si se respetaban las disposiciones regias, tanto en el Colegio Mayor de San Ildefonso como los distintos colegios menores de la Universidad de Alcalá. Medrano desarrolló además una importante labor de supervisión sobre el estado de los edificios universitarios, algunos de ellos en condiciones casi de ruina, por lo que demandaba una urgente actuación.

El Colegio de las Santas Justa y Rufina sobrevivió a la reforma de Medrano, pero dentro ya de una dinámica de decadencia que, por otra parte, era ya algo común a muchos colegios menores vinculados a la Universidad de Alcalá. Las causas estuvieron en su inmovilismo, el temor a la novedad; sus objetivos teológicos; su metodología escolástica, por la relajación de la disciplina, con el incumplimiento de las elementales obligaciones docentes y de los preceptos constitucionales; por los abusos en las certificaciones y colaciones de grados; y por la pésima situación financiera en la que vivían permanentemente instalados los colegios.

Algo que se constataba en 1753, ya que, según el Catastro de Ensenada, y antes del inicio del proceso de agregaciones colegiales que afectaron al Colegio de las Santas Justa y Rufina, el colegio contaba tan sólo con dos becas y un familiar ${ }^{6}$, cuando había sido fundado para una dotación de diez becas y tres familiares.

\section{Las críticas de los ilustrados}

Sobre esta problemática muy pronto escribieron las grandes plumas de la Ilustración española, desde Gaspar Melchor de Jovellanos ${ }^{7}$ a Pedro Murillo Velarde ${ }^{8},-$ los dos habían estado en la Universidad de Alcalá-, pasando por

${ }^{6}$ Alcalá de Henares, 1753. Según las Respuestas Generales del Catastro de Ensenada. Introducción de Arsenio Lope Huerta. (Alcabala del Viento. Tabapress. Madrid, 1992), p. 120. Otero, Luis E., Carmona, Carlos y Gómez, Gutmaro: La ciudad oculta: Alcalá de Henares, 1753-1868. El nacimiento de la ciudad burguesa. (Alcalá de Henares. 2003), p. 101. Calleja, José D., Obras completas. (Alcalá de Henares, 2000).

${ }^{7}$ Gutiérrez, Luis M. "Gaspar Melchor de Jovellanos (1744-1811)”. (En Gutiérrez, Luis M., Casado, Manuel y Ballesteros, Pedro: Profesores y estudiantes. Biografía colectiva de la Universidad de Alcalá (1508-1836). Alcalá de Henares. Universidad de Alcalá. 2013).

${ }^{8}$ Serrera, Ramón Mạ. "Estudio preliminar". (En Murillo, Pedro: Geographía de América (1752). Granada. Universidad de Granada. 1990, pp. XIII-XLII Casado, Manuel: "Pedro Murillo Velarde y Bravo (1696-1753)". (En Gutiérrez, Luis M., Casado, Manuel y Ballestero, Pedro: 
Francisco Pérez Bayer ${ }^{9}$, Gregorio Mayans, el padre Feijoo o Pablo de Olavide, entre otros. Y todos ellos, a la hora de buscar a los culpables de los males de la universidad, coincidían en acusar a dos instituciones. Una, la Compañía de Jesús, que había volcado su poder e influencia para controlar la educación a todos los niveles, y que acabaría siendo objeto de expulsión de los territorios hispanos. Y la otra, cómo no, la institución colegial, representada por los seis colegios mayores, los cuatro de Salamanca, el de Valladolid y el de San Ildefonso de Alcalá, que muy pronto serían receptores de las más duras críticas y objeto de reforma, en una dinámica de agrio enfrentamiento para intentar acabar con un "poder colegial" perfectamente configurado ${ }^{10}$.

En el caso del Colegio Mayor de San Ildefonso de Alcalá se le reprochaba el haber formado una estrecha simbiosis con el conjunto de la Universidad de Alcalá, a la que controlaba. Sus colegiales constituían un verdadero núcleo de poder, prácticamente autónomo y con un fuerte espíritu corporativista, lo que les permitía estar coligados entre ellos para repartirse los mejores puestos de gobierno y administración, tanto civil como eclesiástica. Colegiales y excolegiales conformaron una casta cerrada que provocaba el recelo y el odio del resto de la comunidad universitaria. Un estatus privilegiado que mantuvieron durante siglos, perpetuando una "académica palanca", similar a la de los colegiales mayores salmantinos ${ }^{11}$, sobre todo los del Colegio Mayor de San Bartolomé, porque supieron articular distintos sistemas de control, desde las mismas adjudicaciones de becas y de cátedras, situación que también padecieron los colegiales del menor de las Santas Justa y Rufina. La razón era obvia, las cátedras constituían el primer paso para arrancar una fructífera carrera administrativa en cualquier ámbito civil o eclesiástico del servicio y entrega a la educación universitaria y a la ciencia.

Profesores y estudiantes. Biografía colectiva de la Universidad de Alcalá (1508-1836). Alcalá de Henares. Universidad de Alcalá. 2013).

${ }^{9}$ Pérez, Francisco. Por la libertad de la literatura española. Estudio preliminar de Antonio Mestre Sanchis. (Alicante. Instituto de Cultura Juan Gil Albert. Diputación de Alicante. 1991). Pérez, Francisco: Diario histórico de la reforma de los seis Colegios Mayores de Salamanca, Valladolid y Alcalá. (Valencia. Generalitat Valenciana. 2002).

${ }^{10}$ Álvarez, Antonio. La Ilustración y la Reforma de la Universidad en la España del Siglo XVIII. Edición conmemorativa del II Centenario de Carlos III. (Madrid. Instituto Nacional de Administración Pública. 1988). Álvarez, Antonio: "El Colegio Mayor de San Ildefonso y la configuración del poder colegial". (En Estudios de historia de la universidad española. Madrid. Ediciones Pegaso, 1993).

${ }^{11}$ Carabias, Ana Ma . "Salamanca, académica palanca hacia el poder". (En Aranda, Francisco J. (Coord.): Letrados, juristas y burócratas en la España moderna. Cuenca. Universidad de Castilla-La Mancha. 2006). 
Pero las mayores críticas contra los colegios mayores y ese poder de la "casta colegial" fueron escritas por Francisco Pérez Bayer, autor que redactó un amplio y riguroso "estado" de la situación general de las universidades, incluida la Universidad de Alcalá, ilustrándolo con diferentes ejemplos sobre el abuso del juego con apuestas por parte de los colegiales, de las contiendas, violencias y tropelías que se producían, y de la vanidad y rebeldía que les caracterizaba ${ }^{12}$.

No podemos detenernos en el tema del poder colegial, bien estudiado para Salamanca o Valladolid y poco o nada para Alcalá, pero sí destacar que el poder que tenían los seis colegios mayores españoles, dentro y fuera de la administración, fue la causa de los múltiples obstáculos con que se realizó el proceso reformista en la universidad en tiempos de Carlos III.

\section{La Universidad de Alcalá en el siglo XVIII}

A este respecto, ya en 1772, el propio Pedro Rodríguez Campomanes, desde su cargo de Fiscal de Consejo de Castilla, y tras analizar en detalle los informes de los claustros de las distintas facultades para la elaboración de un nuevo Plan de Estudios, reconocía "la decadencia en que se hallaba la enseñanza en aquella célebre universidad que era la de Alcalá". Campomanes, como otros, fue consciente de que no sólo era necesaria la reforma de los planes de estudio o descartar asignaturas inútiles, sino que era necesario algo de más calado, como era acabar con "ciertos abusos y corruptelas que con el tiempo se habían introducido". Sin embargo, los esfuerzos reformistas resultaron en gran medida infructuosos, haciendo imposible "recuperar aquella grandeza que permitió ver florecer en esta universidad la erudición y sabiduría a un tiempo" ${ }^{13}$.

Si la situación de las universidades españolas no era nada buena a principios del siglo XVIII, en el caso de Alcalá era más grave si cabe por el enfrentamiento con los colegios menores seculares, que cuestionaban la autoridad del Colegio Mayor de San Ildefonso por sus reiterados abusos; por la abierta vía para la destrucción y expolio de su patrimonio; por los desacuerdos con el cabildo de la Iglesia Magistral ${ }^{14}$; por los conflictos con las órdenes

${ }^{12}$ Pérez, Francisco. Diario histórico de la reforma de los seis Colegios Mayores de Salamanca, Valladolid y Alcalá. (Valencia. Generalitat Valenciana. 2002).

${ }^{13}$ Gutiérrez, Luis M. y Ballesteros, Pedro. Cátedras y Catedráticos de la Universidad de Alcalá en el siglo XVIII. (Alcalá de Henares. Universidad de Alcalá. 1998), p. 28.

${ }^{14}$ Gutiérrez, Luis M. "Relaciones institucionales entre el Colegio Mayor de San Ildefonso de la Universidad de Alcalá y las autoridades eclesiásticas del Arzobispado de Toledo: tensiones y conflictos en la Edad Moderna". Anales Complutenses. Volumen XV (2013). 
religiosas instaladas en Alcalá, titulares de varios colegios menores de regulares; y por la rápida y acusada disminución de la matrícula universitaria. En definitiva, por la ruina económica generalizada a causa de una pésima administración de las rentas ${ }^{15}$.

Ante este cúmulo de problemas descrito por autores como los citados, la actitud del Colegio Mayor de San Ildefonso fue la de aferrarse una vez más a sus innumerables privilegios y permanecer así. Pero, desde mediados del siglo XVIII, ya se encontraba aislado, teniendo enfrente al resto de miembros que conformaban la universidad, como señalaba Vicente de la Fuente ${ }^{16}$, un hombre muy crítico con esa "tiranía" del Colegio Mayor de San Ildefonso, y sus efectos negativos para el conjunto de la vida universitaria. Tiranía, prepotencia colegial o "meritocracia", frente a la cual se aliaron todos los agraviados y descontentos, para combatir los abusos, el descuido de algunas cátedras, y, en definitiva, la grave decadencia de la universidad.

Esa hostilidad creciente hacia el Colegio Mayor de San Ildefonso por parte de los colegios menores, seculares y regulares, de una buena parte del profesorado, de los manteístas y de destacados intelectuales de la época, apuntaba ya ciertos signos de debilidad de la poderosa institución colegial mayor. El propio Vicente de la Fuente hablaba de la "anarquía cismática de Alcalá", donde él había sido colegial en su juventud ${ }^{17}$.

\section{La reforma de Díaz de Rojas}

En este escenario es en el que tuvo que sobrevivir el Colegio de las Santas Justa y Rufina, cuyos colegiales se vieron alejados de becas, cátedras y cargos, hasta que la situación empezó a cambiar en la década de 1760 cuando se pusieron en marcha unas medidas reformistas ${ }^{18}$ que, finalmente, acabarían con la hegemonía del Colegio Mayor de San Ildefonso. Sin embargo, el resul-

${ }^{15}$ Gutiérrez, Luis M. "La Universidad de Alcalá: apuntes para una Historia” (En La Universidad de Alcalá II. Madrid. Colegio Oficial de Arquitectos de Madrid. 1990), tomo II.

${ }^{16}$ Gutiérrez, Luis M. "Vicente de la Fuente (1817-1889)". (En Gutiérrez, Luis M., Casado, Manuel y Ballesteros, Pedro: Profesores y estudiantes. Biografía colectiva de la Universidad de Alcalá (1508-1836). Alcalá de Henares. Universidad de Alcalá. 2013), 239-241.

${ }^{17}$ Fuente, Vicente de la. Historia de las Universidades, Colegios y demás establecimientos de enseñanza en España. (Madrid. Imprenta de la Viuda e Hija de Fuentenebro. 1889).

${ }^{18}$ Colección de Reales Ordenes, y Providencias dadas por S.M. y su Supremo Consejo, en razón de la enseñanza, y gobierno de la Universidad de Alcalá de Henares desde 1760. Impresa en virtud de Reales Órdenes del Consejo, por los originales quedan en la Secretaría de dicha Universidad. Alcalá. Imp. María Espartosa y Briones, 1773. 
tado de las reformas para muchos colegios menores como el de las Santas Justa y Rufina sería la agregación a otro colegio, en su caso el de Santa Catalina Mártir o de los Verdes.

En efecto, fue el cancelario y visitador Pedro Díaz de Rojas, quien estuvo detrás de la serie de reales cédulas que modificarían la esencia de la Universidad de Alcalá al intervenir directamente sobre el Colegio Mayor de San Ildefonso. Un ilustrado en quien coincidían la amistad con Manuel de Roda y Arrieta y su condición de miembro del Consejo de la Suprema y General Inquisición, además de Cancelario universitario y Juez Académico y Apostólico de la Universidad de Alcalá.

Pedro Díaz de Rojas fue antiguo colegial del Colegio de San Ciriaco y Santa Paula o de Málaga, doctor en Cánones por Alcalá y juez del Consejo de la Gobernación del arzobispado de Toledo desde el 29 de octubre de $1767^{19}$. En 1774 el cardenal Lorenzana le había nombrado Vicario General de la Audiencia de Alcalá y de todo el arzobispado de Toledo y Capellán Mayor de la Magistral. En 1775, quedar vacante la Abadía de la Magistral y Cancelario de la universidad, le propuso para ocupar dicha dignidad. Carlos III ya le había designado "para entender en la Comisión especial respectiva al Colegio mayor de la Universidad de dicha ciudad"20.

En agosto de 1776 cesó el Rector del Colegio Mayor de San Ildefonso y Universidad de Alcalá, Sancho Llamas y Molina ${ }^{21}$ y el 8 de octubre de 1776, Pedro Díaz de Rojas ya aparece en los Libros de Actos y Grados como Rector, Abad Mayor, Canciller y Juez Académico de la Universidad de Alcalá22, ostentando toda la auctoritas y la potestas de la universidad.

El día 10 mayo de 1777 el Secretario de Estado y del Despacho de Gracia y Justicia, Manuel de Roda y Arrieta, le trasladó la correspondiente Real Orden para el arreglo y reforma de los colegios menores de la Universidad de Alcalá ${ }^{23}$. A pesar de ciertas reticencias iniciales, Díaz de Rojas aceptó el co-

${ }^{19}$ Relación de los títulos, méritos, grados y exercicios literarios del Doctor don Pedro Díaz de Roxas, Juez del Consejo de la Gobernación de Toledo, Madrid, 19 diciembre 1770. AGS. Simancas, Valladolid. Gracia y Justicia, leg. 119.

${ }^{20}$ Memorial de Díaz de Roxas al rey. Madrid 3 diciembre 1775. AGS. Simancas, Valladolid. Gracia y Justicia, leg. 119, mss.

${ }^{21}$ Necrológica de Sancho Llamas y Molina. Gaceta de Madrid, 22 septiembre de 1829, p. 501. Aznar, Ramón. "La bondad del juez: la mejor garantía de justicia. Un discurso de Sancho de Llamas y Molina (1797)”. Anuario de Historia del Derecho Español. Tomo LXXX (2010), 551-555.

${ }^{22}$ Actos y Grados, 8 octubre 1786. AHN. Madrid. Universidades, lib. 409.

${ }^{23}$ Reales resoluciones comunicadas por el Secretario de Estado de Gracia y justicia, D. Manuel de Roda, al visitador y rector de la Universidad de Alcalá D. Pedro Díaz de Rojas, cancelario de la Universidad, y providencias de éste. AHN. Madrid. Universidades, leg. 549, exp. 3. 
metido, si bien pasarían varios años hasta que la reforma se hiciera efectiva. Entre 1777 y 1783 se vivieron graves conflictos derivados de sus actuaciones, enfrentamientos con los colegiales mayores y menores, y con profesores significados que encontraron en el claustro un espacio para sus protestas, que trascendieron el ámbito universitario, implicando a la propia ciudad de Alcalá de Henares.

Pero también es cierto que la clave de todo el proceso vendría de la mano de los nuevos Estatutos para el Colegio Mayor de San Ildefonso, otorgados por Real Cédula de 21 de febrero de $1777^{24}$, y en los que se recopilaban todos los antecedentes legislativos, como la Real Cédula de 23 de febrero de 1771 por la que se renovaban las Constituciones, con señalamiento de penas a su incumplimiento. Una Real Cedula dirigida al Colegio Mayor de San Ildefonso, de 3 de marzo de 1771, hacía referencia expresa a esa inobservancia, y a otros temas, como la limitación de las "hospederías" a un año o la prohibición de la intervención de "hacedores" para la provisión de becas, mostrando la decadencia en la que se hallaba la Universidad.

En definitiva, con el nuevo texto de los Estatutos se pretendían corregir los males universitarios, pero a la vez se desvirtuaba el espíritu fundacional del propio Cisneros a decidirse la separación del Colegio Mayor de San Ildefonso del resto de la Universidad de Alcalá.

Por otra parte, hasta el 7 de octubre de 1780 no presentó Díaz de Rojas su plan para la reforma de los colegios menores de la Universidad de Alcalá. El 6 de mayo de 1781 el Inquisidor General y obispo de Salamanca, Felipe Beltrán Serrano lo aprobó. Y el 7 de julio otra Real Orden autorizó la Unión de los Colegios. Los colegios de San Jerónimo o Trilingüe ${ }^{25}$, de Santa María de Regla y San Justo y Pastor o de León y de San Martín y Santa Emerenciana o de Aragón ${ }^{26}$ se incorporaron al Colegio de San Ciriaco y Santa Paula o de Málaga. Pero el 14 de julio de 1781, otra Orden del Secretario de Gracia y Justicia, con aprobación regia del 27 del mismo mes, dictó un nuevo Auto de

${ }^{24}$ AHN. Madrid. Universidades, lib. 1143-F. Gutiérrez, Luis M.: “Edición de los Estatutos del Colegio Mayor de San Ildefonso de 1777". Anales Complutenses, VIII (1996).

${ }^{25}$ Capilla colegial para protestar sobre la unión. AHN. Madrid. Universidades, lib. 1001. González, Ramón: "El Colegio Trilíngüe o de San Jerónimo. Aproximación a la historia de sus comienzos en el siglo XVI". (En Actas del III Encuentro de Historiadores del Valle del Henares. Guadalajara. Institución Marqués de Santillana. 1992). Ballesteros, Pedro: "El Colegio de San Jerónimo o Trilingüe", (En Valle, José Luis (Coord.), Colegios Menores Seculares de la Universidad de Alcalá. Alcalá de Henares. Institución de Estudios Complutenses. 2012).

${ }^{26}$ Acta de Unión de Colegios. AHN. Madrid. Consejos, leg. 5492. Ruiz, Ignacio, Alonso, Pedro Manuel y Casado, Manuel: El Colegio de Aragón de la Universidad de Alcalá de Henares. Contexto Histórico y Jurídico (1611-1779). (Madrid. Editorial Dykinson. 2001). 
Unión, esta vez del Colegio de San Clemente Mártir o de los Manchegos y el de las Santas Justa y Rufina, con el Colegio de Santa Catalina Mártir o de los Verdes, nombrando nuevos rectores.

Se dotó al colegio con 14 becas; una por el de las Santas Justa y Rufina; seis por el de San Clemente, dos del patrono y cuatro de libre elección; y siete por el propio colegio de Santa Catalina Mártir o de los Verdes, de ellas dos de Juana Gamboa, una por la familia fundadora y una por cada uno de los condes de Coruña, doña Gracia de Atocha, Presidente del Consejo de Castilla y Juan Bautista de Ochoa, dictándose nuevas Constituciones en $1788^{27}$.

Así, la reforma de Díaz de Rojas de los colegios seculares de la Universidad de Alcalá supuso que éstos se viesen reducidos los Colegios a cinco: el de San Ciriaco y Santa Paula o de Málaga, a partir de ahora Colegio Teólogo de Málaga; el de Santiago o de los Caballeros Manriques; el de San Felipe y Santiago o del Rey; el de Santa Catalina Mártir o de los Verdes; y el nuevo de la Inmaculada Concepción, cuyas constituciones son de $1779^{28}$.

Este fue el resultado de la actuación de Díaz de Rojas, tras remitir su informe final al rey, el 7 de octubre de 1780. Dicho informe fue compuesto a partir de los "cuadernos" levantados para cada colegio menor visitado, reconociendo sus estatutos, acuerdos, estado de las rentas, gobierno de cada uno, etc. Una documentación exhaustiva con todas las irregularidades que presentaban por la reiterada violación de sus constituciones colegiales, comportándose de un modo muy alejado de los deseos de sus fundadores y patronos.

Cuando murió, gran parte de su obra de visitador se deshizo, volviéndose a antiguos usos, formas y comportamientos, y a la falta de dirección y objetivos claros. El panorama se completaba con la ruina económica, el reducido número de alumnos y la progresiva instalación de cuarteles militares en la ciudad de Alcalá de Henares. Todos ellos, factores determinantes para que el siglo XVIII acabase para la Universidad de Alcalá peor aún de lo que había comenzado ${ }^{29}$.

No tardaría en llegar la Real orden de 27 de abril de 1780 por la que se refundían "los colegios de Teólogos de la Madre de Dios, Santa Catalina o los Físicos, Santa Balbina y los Lógicos (que comprendía ya los antiguos de San Isidoro y San Eugenio), San Ambrosio y Trilingüe en uno llamado de la Inmacu-

\footnotetext{
${ }^{27}$ Constituciones del Colegio de Santa Catalina Mártir, 1788. AHN. Madrid. Consejos, leg. 5494.

${ }^{28}$ Constituciones del Colegio de la Inmaculada Concepción, 1779. AHN. Madrid. Consejos, leg. 5492.

${ }^{29}$ Álvarez, Antonio. La Ilustración y la Reforma de la Universidad en la España del Siglo XVIII. Edición conmemorativa del II Centenario de Carlos III. (Madrid. Instituto Nacional de Administración Pública. 1988), 227.
} 
lada Concepción para 62 colegiales pobres con limpieza de sangre instalado en el edificio del de la Madre de Dios. Se comisionó al Abad Mayor de la Magistral y Cancelario de la Universidad para la administración y gobierno del colegio según las Constituciones que se imprimieron".

La actuación del visitador Díaz de Rojas, aunque siguiendo la reforma planteada para el resto de las universidades españolas, tuvo al menos tres aspectos que le particularizan en Alcalá de Henares.

En primer lugar, destacó por el desconocimiento que sobre la organización de la misma tenían los propios reformadores, y en ese sentido hubo mucha improvisación. Nada que ver, por ejemplo, con la llevada a cabo en Salamanca por el obispo Felipe Beltrán Serrano ${ }^{30}$. Después la realidad del hecho fundacional y efectivo de la identificación entre el Colegio Mayor de San Ildefonso y la Universidad de Alcalá, con un mismo Rector para ambas instituciones. Y, por último, la manifiesta decadencia por la que atravesaba.

La reforma avanzó por la fórmula de la aplicación de las reales cédulas relativas al Colegio Mayor de San Ildefonso. Se decidió la de expulsión de sus colegiales y la "repoblación" por otros, aunque éstos mostraron una actitud similar a la de los precedentes y fueron igualmente expulsados. Se aprobaron nuevos y modernos planes de estudios. Se crearon las figuras de director y censor regio. Se intervino el sistema para la obtención de grados. Se atajaron los abusos en la provisión de cátedras. Y, en general, hubo un mayor control por parte del Consejo de Castilla.

Como balance de la reforma nos queda la idea de que "la resistencia opuesta por el partido colegial a la reforma, aunque no logró evitarla, pues los reformadores consiguieron ganar la voluntad del rey, sí que, en definitiva, lograron que fracasara, ya que con su oposición la llevaron a que tomara la reforma un aire de revancha clasista por parte de los reformadores, que ha llevado a ver que la 'liberación' de los Colegios Mayores aparezca mediatizada, de un lado, en función de los intereses regalistas, y, de otro, por los sentimientos sectarios del bando anticolegial. Es cierto que al sectarismo de los colegiales algunos de los reformadores opusieron un sectarismo de signo contrario"31.

${ }^{30}$ Sala, Luis. Visitas y reformas de los Colegios Mayores de Salamanca en el reinado de Carlos III. (Valladolid. Universidad de Valladolid. 1958). Peset, Mariano y Peset, José Luis: El reformismo de Carlos III y la Universidad de Salamanca. Plan general de estudios dirigido a la Universidad de Salamanca por el Real y Supremo Consejo de Castilla en 1771. (Salamanca. Universidad de Salamanca. 1969).

${ }^{31}$ Álvarez, Antonio. La Ilustración y la Reforma de la Universidad en la España del Siglo XVIII. Edición conmemorativa del II Centenario de Carlos III. (Madrid. Instituto Nacional de Administración Pública. 1988), 230. 
En los nuevos planes universitarios todos los colegios debían ser reducidos a una posición secundaria dentro de la universidad, como "seminarios de estudios" dependientes de la misma. La idea acabó fracasando, y la propia reforma, tras producir un primer efecto positivo al suprimir el poder de los colegios, no consiguió que prosperaran las nuevas instituciones puestas en su lugar. Los colegios caminaron hacia su extinción definitiva. Aunque las medidas reformistas no se cumplieron y, por acertadas que fuesen, mal podían sacar a la Universidad de Alcalá de su decadencia.

Además, desde el mismo Consejo de Castilla se siguió una política opuesta a la reforma iniciada, ya que se concedieron privilegios a unos seminarios diocesanos que tampoco salvaron la situación. Realmente, tras el ímpetu inicial de la reforma, pasados veinte años, ninguna medida de las adoptadas había afectado en lo más mínimo a la situación tradicional de la universidad en materia de gobierno, jurisdicción, etc., quedando reducida a la mera existencia de un nuevo plan de estudios ${ }^{32}$. Y puede decirse que, a fin de cuentas, había fracasado tanto la reforma de los colegios, como la de la universidad propiamente dicha, siendo en este contexto reformista general y universitario, y particularmente el vivido en Alcalá de la mano de Díaz de Rojas, en el que llevó a la agregación del Colegio de las Santas Justa y Rufina al de Santa Catalina Mártir o "de los Verdes" ${ }^{33}$ a raíz de iniciar el citado cancelario y visitador su actuación en 1779. Por una situación similar atravesaron el Colegio de San Clemente o "de los Manchegos" ${ }^{34}$ con sus propios agregados, el de San Cosme y San Damián o "de Mena" ${ }^{35}$, que lo había sido en 1759, y el de San Lucas Evangelista o "de Magnes" ${ }^{36}$.

${ }^{32}$ Real Provisión del Consejo, que comprehende el plan de estudios, que ha de observar la Universidad de Alcala de Nares (sic). En Madrid en la imprenta de Pedro Marín, 1772. También contiene la Real Provisión de las declaraciones con que la Universidad ha de observar el Plan de Estudios. Peset, Mariano y Mancebo, Pilar: "Carlos III y la legislación sobre universidades". Documentación Jurídica, 57 (enero-mayo, 1988).

${ }^{33}$ Casado, Francisco J. El Colegio de Santa Catalina Mártir o de los Verdes de la Universidad de Alcalá de Henares: Cuatro siglos de historia de una institución autónoma y su edificio (15861992). (Alcalá de Henares. 1992).

${ }^{34}$ Ruiz, Ignacio. Fuero universitario y Constituciones del Colegio de San Clemente de la Universidad de Alcalá. Contexto histórico, estudios y documentos. (Madrid. 1999).

${ }^{35}$ Gutiérrez, Ma Pilar y Casado, Francisco J. "Fundaciones universitarias españolas libres de patronato regio o eclesiástico. Los colegios autónomos de Alcalá de Henares fundados en el siglo XVI: San Cosme y San Damián o de Mena y Santa Catalina Mártir de los Verdes". (Actas del I Encuentro de Historiadores del Valle del Henares. Guadalajara. 1988).

${ }^{36}$ Sánchez, Manuel V. "Colegio de San Lucas Evangelista o de Magnes". (En Valle, José Luis (Coord.): Colegios menores seculares de la Universidad de Alcalá. Alcalá de Henares. Institución de Estudios Complutenses. 2012). 
Corrían tiempos de reforma y estos colegios menores acabaron adscritos y unificados en el Colegio de Santa Catalina Mártir o de los Verdes, que era el que, a juzgar por los artífices de la reforma, reunía las mejores condiciones para tratar de superar la fuerte crisis económica por la que atravesaba la Universidad de Alcalá. En tal sentido, el propio visitador, que continuó agrupando colegios, vendería a Isidro Lizana el edificio del Colegio de las Santas Justa y Rufina, cuando todavía detentaba el cargo de Rector del Colegio Mayor de San Ildefonso, presumiblemente en calidad de administrador-apoderado.

La intervención del visitador Díaz de Rojas en el Colegio de Santa Catalina Mártir o de los Verdes provocó un largo pleito, de 1781 a 1788, con el Rector y parte de los colegiales verdes, quienes contaban con el apoyo y protección de la patrona del colegio y de algunos miembros del consejo eclesiástico de la ciudad. Al parecer hubo excesos de los colegiales, falta de acatamiento a las disposiciones del visitador, y por parte de este, algunos graves errores formales a la hora de tratar con la instancia colegial, lo que motivó la intervención del Consejo de Castilla para tratar de dilucidar las causas de tan enconado enfrentamiento.

Finalmente, el visitador Pedro Díaz de Rojas sería jubilado por el ministro Floridablanca, y las "nuevas" constituciones del Colegio de Santa Catalina Mártir o de los Verdes aparecerán firmadas por su sucesor, Juan Lucas López ${ }^{37}$.

Pleitos y reformas no consiguieron impedir que los colegiales mayores detentaran el monopolio de las becas, de los órganos de gobierno y de las cátedras, siendo la autoridad del rector la imperante, frente a los claustros de las facultades, los colegios menores y los manteístas. La serie de reales cédulas emitidas desde 1750 buscando recuperar un pasado inexistente tampoco podían ocultar los numerosos problemas de porcionistas, hospederías, clausura y honestidad. Ni el hecho de que algunos de los colegiales mayores y menores, terminados sus años de beca permanecían en los colegios a la espera de algún cargo. La realidad es que pasaban algunos meses hasta conseguir una cátedra, una canonjía o un cargo de oidor o fiscal en alguna audiencia peninsular o americana. En muchos casos, esa espera era de años, en los cuales se realizaban sustituciones de cátedra y se trabajaba para perpetuar el control que ejercía el Colegio Mayor de San Ildefonso al amparo de la larga experiencia universitaria acumulada.

${ }^{37}$ Ejemplar de las Constituciones del Colegio de Santa Catalina Mártir o de los Verdes (se corresponden con las de 1763). En este mismo legajo hay también un ejemplar de las Constituciones del Colegio Mayor de San Ildefonso del año 1715, impreso por García de Briones y que incluye las reformas de García de Medrano, que se pueden confrontar con el contenido del legajo 1142-F de la sección de Universidades del citado Archivo. AHN. Madrid. Consejos, legajo 5494, no 19.1788. 
Así fue como llegó a su final el Colegio de las Santa Justa y Rufina, agregado al de Santa Catalina Mártir o de los Verdes ${ }^{38}$, que se convirtió este, sin duda, en el mayor receptor de colegios reformados, más incluso que el Colegio Teólogo de Málaga ${ }^{39}$.

A partir de 1788 el reformador Díaz de Rojas fue sustituido por Juan de Lucas López, miembro del claustro de la Universidad y arcipreste de la Iglesia Magistral, nombrado por el Conde de Floridablanca, con funciones ampliadas en enero de 1792 por el nuevo Ministro de Gracia y Justicia, Antonio Porlier y Sopranis, Marqués de Bajamar, quien había sido antiguo estudiante de Derecho Civil en la Academia de San José de la Universidad de Alcalá ${ }^{40}$.

En el ejercicio de su cometido, Juan de Lucas López no sólo realizó un proyecto fallido de constituciones generales de la Universidad, sino que también redactó aquéllas por las que se habría de regir a partir de entonces el Colegio de los Verdes, así como también las nuevas para el Colegio Teólogo de Málaga, ambas fechadas en 1788. Pero el desfase era muy grande y la fórmula de unas nuevas constituciones no era la más apropiada para reconducir la situación de deterioro a la que se había llegado, por lo que las reformas fueron un intento fallido para encauzar a los colegios ante su forma lamentable de vida, intentando recuperar además estudiantes y rentas.

El Colegio de las Santas Justa y Rufina sólo disponía de una beca, para estudiantes sevillanos, de las diez colegiaturas con las que había sido dotado según las constituciones fundacionales, quedando reflejados para la posteridad los datos la fundación de este colegio, años después, en 1789, en el:

"Memorial ajustado hecho con citación y asistencia de las partes en virtud de decreto del Consejo pleno, del pleito que se sigue en él a consulta con S.M. por la Condesa de Baños, como patrona del Colegio de Santa Catalina Mártir, llamado de los Verdes de la Universidad de la ciudad de Alcalá de Henares, y el mismo colegio con el Dr. D. Francisco Xavier Sainz de la Escalera,

${ }^{38}$ Noticia de la Fundación, Progresos y Estado actual de los Colegios de Santa Catalina Mártir de los Verdes y San Ciriaco y Santa Paula de Málaga de esta Real Universidad [de Alcalá de Henares]. AMAH. Alcalá de Henares. División Histórica. Sección Universidades. Legajo 1097/1. Siglo XVIII. Corresponde a una real resolución de 27 de julio de 1781 en la que se recoge la historia del colegio hasta ese momento y resume las innovaciones del reformador García de Medrano.

${ }^{39}$ Gutiérrez, Luis M. y Ballesteros, Pedro. Fuentes Históricas de Alcalá de Henares: textos constitucionales (s. XVIII) y de graduados universitarios (1776-1836). (Alcalá de Henares. Universidad de Alcalá. 2011). Se transcriben las "nuevas" constituciones (1788) del Colegio Teólogo de Málaga.

${ }^{40}$ Hernández, Manuel V. “Antonio Porlier y Sopranis, Marqués de Bajamar”. (En Gutiérrez, Luis M., Casado, Manuel y Ballesteros, Pedro: Profesores y estudiantes. Biografía colectiva de la Universidad de Alcalá (1508-1836). Alcalá de Henares. Universidad de Alcalá. 2013). 
su rector, e individuo, con el Doctor Don Pedro Diaz de Rojas, abad de la Santa Iglesia Magistral de la misma ciudad, en calidad de Visitador de S.M. para el arreglo y reforma de los Colegios de la misma Universidad: sobre excesos de esta comisión, agravios del despojo hecho a dicha Condesa de sus regalías, al dicho Sainz de su Rectorado, y demás puntos contenidos en las diversas pretensiones de las partes" ${ }^{\prime \prime}$.

Un memorial que alude a lo dispuesto en las constituciones en materia de provisión de colegiaturas, y cita el auto del visitador por el cual se unió al "colegio de los Verdes el de Santa Justa y Rufina, y le asignó una Colegiatura, que había de presentar el Patrono, con preferencia para la obtención de dicha Colegiatura a los naturales del Arzobispado de Sevilla" ${ }^{\prime 42}$.

\section{Conclusiones}

A finales del siglo XVIII, pocos eran los colegios menores que quedaban del casi medio centenar que se habían ido fundando en torno al Colegio Mayor de San Ildefonso y Universidad de Alcalá. Una pléyade de colegios seculares, regulares o con otro mecenazgo y patronato, que habían ido surgiendo a lo largo de los siglos XVI y XVII en la estela de la primitiva idea del Cardenal Cisneros de servir para cubrir las etapas previas al ingreso en las facultades de la Universidad de Alcalá.

${ }^{41}$ Memorial ajustado... Del pleito que se sigue... Por la Condesa de Baños, como patrona del Colegio de Santa Catalina Mártir, llamado de los Verdes de la Universidad de... Alcalá de Henares, y el mismo colegio con... D. Francisco Xavier Sainz de la Escalera, su rector..., con... Don Pedro Diaz de... Colaborador Joaquín Ibarra, Biblioteca Complutense (Alcalá de Henares). Publicado por en la Imprenta de la Viuda de Don Joachin Ibarra, 1789, fols. 19v-20v. Universidad Complutense de Madrid (UCM). Madrid. Biblioteca Histórica "Marqués de Valdecilla". Fondo Antiguo. Referencia: BH-FOA-279. Existe una adición a este Memorial ajustado... publicada al año siguiente, en 1790. CSIC. Madrid. Biblioteca: M-Resid. Colección: MP3. Referencia: R571. Adición al Memorial ajustado hecha en virtud de decreto del Consejo... En el expediente consultivo a S.M. Sobre excesos de visitas, y reforma del Colegio de Santa Catalina Mártir, llamado de los Verdes, y demás en la Universidad de Alcalá de Henares. Autor Lorenzo Guardiola y Sáez. Universidad de Alcalá de Henares. Publicado por vda. de Ibarra, 1790, con la respuesta de los tres fiscales que intervienen en el pleito.

${ }^{42}$ Memorial ajustado... Del pleito que se sigue... Por la Condesa de Baños, como patrona del Colegio de Santa Catalina Mártir, llamado de los Verdes de la Universidad de... Alcalá de Henares, y el mismo colegio con... D. Francisco Xavier Sainz de la Escalera, su rector..., con... Don Pedro Diaz de... Colaborador Joaquín Ibarra, Biblioteca Complutense (Alcalá de Henares). Publicado por en la Imprenta de la Viuda de Don Joachin Ibarra, 1789, fol. 20v. UCM. Madrid. Biblioteca Histórica "Marqués de Valdecilla". Fondo Antiguo. Referencia: BH-FOA-279. 
El resultado final fue el reagrupamiento de colegios, siendo precisamente el Colegio Teólogo de Málaga y el de Santa Catalina Mártir o de los Verdes los principales receptores de las instituciones colegiales que se extinguían. Al Colegio de las Santas Justa y Rufina, agregado al de Santa Catalina Mártir o de los Verdes, por su nueva situación y condición, le tocó vivir los enfrentamientos que se produjeron entre los colegios y sus representantes, con el visitador Díaz de Rojas, abriéndose toda suerte de pleitos y situaciones de verdadera rebeldía. El resultado fue un enorme desgaste de la institución colegial, que no pudo atajarse con las reformas, ni con la redacción de unas nuevas constituciones generales para la Universidad de Alcalá, las de 1788, que nunca llegaron a ser aprobadas ni pudieron entrar en vigor de manera definitiva.

Sabemos de ese largo proceso de desintegración del sistema colegial por la documentación acumulada en los pleitos planteados por de los herederos de los fundadores, tanto del Colegio de Santa Catalina Mártir o de los Verdes como del Teólogo de Málaga. Ambos colegios se sintieron engañados y robados, porque no se cubrían las becas y se disputaban el patrimonio de los colegios incorporados. Unos pleitos que continuaron con el visitador Juan de Lucas López, a partir de 1790.

Este ocaso colegial fue el que posibilitó el desmantelamiento de la Universidad de Alcalá. Además del Colegio Teólogo de Málaga y el de Santa Catalina Mártir o de los Verdes, sólo podemos citar como supervivientes a otros tres colegios menores: el de los Irlandeses, incorporado en 1785 a su homónimo, aunque Mayor, de Salamanca; el de San Felipe y Santiago o del Rey, quedando excluido de las medidas reformistas de Juan de Lucas y López, su sucesor; y el de los Caballeros Manriques, visitado ya en 1778 por Díaz de Rojas. Al final, unos y otros, vieron incautados sus bienes y suprimidos en 1843, produciéndose el cierre definitivo de la Universidad y su traslado definitivo a Madrid.

Para ir concluyendo, tras tres siglos de andadura, aquel novedoso "modelo" colegial de Alcalá, también trasladado a América, se había agotado. A su fin contribuyeron las últimas actuaciones de unos rectores perpetuos y colegiales huéspedes, que se habían señoreado de sus propias instituciones colegiales, cuando la mala administración y la falta de rentas les habían dejado las manos libres para hacerlo. Baste con recordar las graves denuncias y acusaciones que al respecto formularon ya algunos lúcidos coetáneos como ya hemos señalado.

En Alcalá de Henares, a pesar de las múltiples vicisitudes acontecidas, han permanecido muchos de los colegios menores, su Iglesia Magistral y el componente ideológico y espiritual plasmado en la ornamentación escultó- 
rica de la fachada del Colegio Mayor de San Ildefonso, Las reformas, los cambios en el plan de estudios, las desapariciones y agregaciones de colegios y el final que esperaba a la universidad, no acabaron con el "poso" de una universidad que había sido algo más, haciendo de Alcalá de Henares un verdadero complejo docente de inspiración eclesiástica, una "civitas dei, civitas complutensis", trasmutada en "ciudad del saber".

Pero nos quedamos con esa Alcalá de Henares y su recinto universitario que, a partir de 1508 fue integrando en su componente jurisdiccional, ideológico y espiritual, tanto la propia Iglesia Magistral y su cabildo, como a toda la pléyade de colegios menores de distinta naturaleza y patronato, entre los que se encontraba el de las Santas Justa y Rufina, en el ámbito del Colegio Mayor y de su "todopoderoso" rector. Ahí radicó su singularidad, en la rotundidad de que era el Colegio Mayor de San Ildefonso y Universidad de Alcalá el artífice de la vertebración y articulación de la "ciudad del saber", definiendo un "modelo" novedoso y de proyección universal.

La trayectoria colegial de Alcalá tuvo su final en 1842. Desde la Universidad Central, en Madrid, se nombró un visitador que cerró los colegios alcalaínos haciéndose cargo de todos sus efectos. El 4 de noviembre de 1843, un decreto del Rector de la misma, el Doctor en Jurisprudencia Pedro Sabau y Larroya, incorporó a ésta sus bienes y rentas.

\section{Bibliografía}

Alcalá de Henares, 1753. Según las Respuestas Generales del Catastro de Ensenada. Introducción de Arsenio Lope Huerta. Madrid. Alcabala del Viento. Tabapress. 1992.

ÁlvAREZ, ANTonio. La Ilustración y la Reforma de la Universidad en la España del Siglo XVIII. Edición conmemorativa del II Centenario de Carlos III. Madrid. Instituto Nacional de Administración Pública. 1988.

Álvarez, Antonio. "El Colegio Mayor de San Ildefonso y la configuración del poder colegial”. En Estudios de historia de la universidad española. Madrid. Ediciones Pegaso, 1993, 29-37.

Álvarez, Antonio. "La decadencia de la Universidad de Alcalá en el siglo XVIII". Estudios de historia de la universidad española. Madrid. Ediciones Pegaso, 1993, 105-106.

AzNAR, RAMóN. "La bondad del juez: la mejor garantía de justicia. Un discurso de Sancho de Llamas y Molina (1797)". Anuario de Historia del Derecho Español. Tomo LXXX (2010), 547-585. 
Ballesteros, Pedro. "El Colegio de San Jerónimo o Trilingüe", En Valle, José Luis (Coord.), Colegios Menores Seculares de la Universidad de Alcalá. Alcalá de Henares. Institución de Estudios Complutenses. 2012. 391.

CALleja, JosÉ D. Obras completas. Alcalá de Henares, 2000, pp. 212-213. CARABIAS, ANA Mà. "Salamanca, académica palanca hacia el poder". En Aranda, Francisco J. (Coord.): Letrados, juristas y burócratas en la España moderna. Cuenca. Universidad de Castilla-La Mancha. 2006, 23-60.

Casado, Francisco J. El Colegio de Santa Catalina Mártir o de los Verdes de la Universidad de Alcalá de Henares: Cuatro siglos de historia de una institución autónoma y su edificio (1586-1992). Alcalá de Henares. 1992, 29-32.

CASADO, MANUEL. "Los colegios menores seculares de patronato eclesiástico de Universidad de Alcalá. Líneas de investigación”. En Rodríguez-San Pedro, Luis E. y Polo, Juan L. (Eds.): Universidades clásicas de la Europa Mediterránea: Bolonia, Coimbra y Alcalá. Salamanca. Miscelánea Alfonso IX, 2005, 209-257.

Casado, Manuel. “Pedro Murillo Velarde y Bravo (1696-1753)". En Gutiérrez, Luis M., Casado, Manuel y Ballestero, Pedro: Profesores y estudiantes. Biografía colectiva de la Universidad de Alcalá (1508-1836). Alcalá de Henares. Universidad de Alcalá. 2013, 459-463.

Casado, Manuel, Alonso, Pedro M. "Colegios menores seculares de patronato eclesiástico y naciones estudiantiles en la Universidad de Alcalá de Henares (siglos XVI-XVII)". Revista de Ciencias de la Educación: Órgano del Instituto Calasanz de Ciencias de la Educación, 212 (2007), 409-456.

Fuente, Vicente DE LA. Historia de las Universidades, Colegios y demás establecimientos de enseñanza en España. Madrid. Imprenta de la Viuda e Hija de Fuentenebro. 1889.

GIL, ÁNGEL. "Consideraciones en torno al reformismo universitario castellano en el siglo XVII". (En Actas del Congreso de Jóvenes Historiadores y Geógrafos. Tomo II. Madrid. 1990), 165-178.

GIL, ÁNGEL. "Reformas en los colegios menores cisnerianos de la Universidad de Alcalá de Henares durante el siglo XVII". (En Actas del III Encuentro de Historiadores del Valle del Henares. Guadalajara. 1992), 369-386.

GIL, ÁNGEL. "Visitas y reformas de la Universidad de Alcalá en el siglo XVII". Anales Complutenses, IV-V (1992-1993), 65-134.

GIL, ÁNGEL. "Reformas de los colegios menores no cisnerianos de la Universidad de Alcalá durante el siglo XVII". Anales Complutenses, VIII (1996), 87-104. 
GIL, ÁNGEL. "Reformas de los colegios menores". En Valle, José L. (Coord.): Colegios menores seculares de la Universidad de Alcalá. (Alcalá de Henares. Institución de Estudios Complutenses. 2012), 341-362.

GonZÁlez, RAMón. "El Colegio Trilíngüe o de San Jerónimo. Aproximación a la historia de sus comienzos en el siglo XVI". En Actas del III Encuentro de Historiadores del Valle del Henares. Guadalajara. Institución Marqués de Santillana. 1992, 231-250.

GutiÉRREZ, Ma Pilar y CASAdo, Francisco J. "Fundaciones universitarias españolas libres de patronato regio o eclesiástico. Los colegios autónomos de Alcalá de Henares fundados en el siglo XVI: San Cosme y San Damián o de Mena y Santa Catalina Mártir de los Verdes". Actas del I Encuentro de Historiadores del Valle del Henares. Guadalajara. 1988, 351-360.

GUTIÉRREZ, LuIS M. "La Universidad de Alcalá: apuntes para una Historia” En La Universidad de Alcalá II. Madrid. Colegio Oficial de Arquitectos de Madrid. 1990, tomo II, 9-89.

GutiéRrez, Luis M. "Edición de los Estatutos del Colegio Mayor de San Ildefonso de 1777". Anales Complutenses, VIII (1996), 139-154.

GutiÉRrez, Luis M. “Vicente de la Fuente (1817-1889)”. En Gutiérrez, Luis M., Casado, Manuel y Ballesteros, Pedro: Profesores y estudiantes. Biografía colectiva de la Universidad de Alcalá (1508-1836). Alcalá de Henares. Universidad de Alcalá. 2013, 239-241.

GutiéRrez, Luis M. "Gaspar Melchor de Jovellanos (1744-1811)”. (En Gutiérrez, Luis M., Casado, Manuel y Ballesteros, Pedro: Profesores y estudiantes. Biografía colectiva de la Universidad de Alcalá (1508-1836). Alcalá de Henares. Universidad de Alcalá. 2013), 344-350.

GuTIÉRREZ, LUIS M. "Relaciones institucionales entre el Colegio Mayor de San Ildefonso de la Universidad de Alcalá y las autoridades eclesiásticas del Arzobispado de Toledo: tensiones y conflictos en la Edad Moderna". Anales Complutenses. Volumen XV (2013), 237-280;

GutiérRez, Luis M. y BALLESTERos, P. Cátedras y Catedráticos de la Universidad de Alcalá en el siglo XVIII. Alcalá de Henares. Universidad de Alcalá. 1998.

Gutiérrez, Luis M. y Ballesteros, P. Fuentes Históricas de Alcalá de Henares: textos constitucionales (s. XVIII) y de graduados universitarios (17761836). Alcalá de Henares. Universidad de Alcalá. 2011.

Hernández, Manuel V. "Antonio Porlier y Sopranis, Marqués de Bajamar". En Gutiérrez, Luis M., Casado, Manuel y Ballesteros, Pedro: Profesores y estudiantes. Biografía colectiva de la Universidad de Alcalá (15081836). Alcalá de Henares. Universidad de Alcalá. 2013, 105-107. 
Martín, Mariano. Estado de la Universidad de Alcalá [1805]. Madrid. Instituto Antonio de Nebrija de Estudios sobre la Universidad. Editorial Dykinson. 1999. 120 pp.

Otero, Luis E., CARmona, Carlos y Gómez, Gutmaro. La ciudad oculta: Alcalá de Henares, 1753-1868. El nacimiento de la ciudad burguesa. Alcalá de Henares. 2003, 101.

PÉRez BAYER, Francisco. Por la libertad de la literatura española. Estudio preliminar de Antonio Mestre Sanchis. Alicante. Instituto de Cultura Juan Gil Albert. Diputación de Alicante. 1991. 597.

PÉREZ BAYER, Francisco. Diario histórico de la reforma de los seis Colegios Mayores de Salamanca, Valladolid y Alcalá. Valencia. Generalitat Valenciana. 2002. 885.

Peset, Mariano y Mancebo, Pilar. "Carlos III y la legislación sobre universidades”. Documentación Jurídica, 57 (enero-mayo, 1988), 208-218.

Peset, Mariano y Peset, José Luis. El reformismo de Carlos III y la Universidad de Salamanca. Plan general de estudios dirigido a la Universidad de Salamanca por el Real y Supremo Consejo de Castilla en 1771. Salamanca. Universidad de Salamanca. 1969.

Ruiz, IgnaCio. Fuero universitario y Constituciones del Colegio de San Clemente de la Universidad de Alcalá. Contexto histórico, estudios y documentos. Madrid. 1999, 69-77.

Ruiz, Ignacio, Alonso, Pedro Manuel y Casado, Manuel. El Colegio de Aragón de la Universidad de Alcalá de Henares. Contexto Histórico y Jurídico (1611-1779). Madrid. Editorial Dykinson. 2001.

SALA, LuIS. Visitas y reformas de los Colegios Mayores de Salamanca en el reinado de Carlos III. Valladolid. Universidad de Valladolid. 1958.

Sánchez, Manuel V. "Colegio de San Lucas Evangelista o de Magnes". En Valle, José Luis (Coord.): Colegios menores seculares de la Universidad de Alcalá. Alcalá de Henares. Institución de Estudios Complutenses. 2012, 225-232.

SERRERA, RAmón Mạ. "Estudio preliminar". En Murillo, Pedro: Geographía de América (1752). Granada. Universidad de Granada. 1990, XIII-XLII.

VAlLe, José L. (Coord.). Colegios Menores Seculares de la Universidad de Alcalá. Universidad de Alcalá. Alcalá de Henares. Institución de Estudios Complutenses. 2012.391. 